\title{
CREACIÓN DE IMAGEN, VISIBILIDAD Y TURISMO COMO ESTRATEGIAS DE CRECIMIENTO ECONÓMICO DE LA CIUDAD
}

\author{
ANTÒNIA CASELLAS ${ }^{1}$ \\ Esteve Dot Jutgla ${ }^{1}$ \\ Montserrat Pallares-Barbera ${ }^{1}$
}

\begin{abstract}
Resumen - El objetivo de este artículo es analizar como el crecimiento económico de Barcelona desde mediados de los años noventa se ha basado en la producción de una economía de servicios avanzados, en el que las estrategias de creación de imagen y el turismo han jugado un papel primordial. En este proceso destaca el papel promotor de macro-eventos como los Juegos Olímpicos de 1992, los cuales han sido herramientas de cambio del modelo económico de Barcelona; y se identifica la importancia de la colaboración público-privada y la planificación estratégica. Se analiza el impacto económico del turismo para la ciudad en el periodo 1990-2010, y se concluye con una reflexión en torno a las implicaciones sobre el espacio público que esta estrategia de crecimiento conlleva.
\end{abstract}

Palabras clave: Barcelona, turismo, desarrollo económico, espacio público, marketing.

Resumo - CRiaÇão de imagem, Visibilidade e turismo. Estratégias de CRESCIMENTO ECONÓMICO COM FUTURO? O objectivo deste artigo é analisar o crescimento económico de Barcelona, desde meados dos anos 90, baseado numa economia de serviços avançados, onde as estratégias de criação da imagem e a dinamização do turismo tiveram papel primordial. Neste processo destaca-se o papel promotor de macro-eventos, como os Jogos Olímpicos de 1992, ferramentas para a mudança do modelo económico de Barcelona, salienta-se a importância da colaboração público-privada e da planificação estratégica e analisa-se o impacto económico do turismo para a cidade no período de 1990-2010. Conclui-se com uma reflexão em torno das implicações sobre o espaço público que esta estratégia de crescimento encerra.

Palavras-chave: Barcelona, turismo, desenvolvimento económico, espaço público, marketing.

Recebido: 12/01/2010. Aceite: 13/07/2010.

1 Departamento de Geografía. Facultad de Filosofía y Letras, Universitat Autònoma de Barcelona. E-mail: antonia.casellas@uab.cat; esteve.dot@uab.cat; montserrat.pallares@uab.cat 


\begin{abstract}
Image CREATIOn, Visibility AND tourism. Growth STRATEgIES FOR THE FUTURE? The aim of this paper is to analyse how Barcelona's economic growth since the 1990s has been based on the production of advanced services, in which image creation and tourism have played a key role. In this context, we emphasise the role played by macro-events, such as the 1992 Olympic Games, which have brought about important changes to Barcelona's economic model, and we highlight the importance of public-private partnerships and strategic planning. Additionally, we analyse the economic impact of tourism upon the city in the period 1990-2010, and we conclude with some reflections on the implications upon public space entailed by this growth strategy.
\end{abstract}

Key words: Barcelona, tourism, economic development, public space, marketing.

\begin{abstract}
Résumé - CRÉATION D'IMAGE, VISIBILITÉ ET TOURISME COMME STRATÉGIES DE CROISSANCE ÉCONOMIQUE DE LA VILLE. L'objectif de cet article est d'analyser comment la croissance économique de Barcelone à partir de la moitié des années 1990 s'est basée sur la production d'une économie de services avancés, au sein de laquelle les stratégies de création d'image et le tourisme ont joué un rôle primordial. Dans ce processus émerge l'importance de la promotion de macro-événements comme les Jeux Olympiques de 1992, lesquels sont considérés comme outils de changement du modèle économique de Barcelone. S'identifient également ici la collaboration public-privée et la planification stratégique. L'article analyse l'impact économique du tourisme dans la ville durant la période 1990-2010, et propose une réflexion sur les implications de l'espace public entraînées par cette stratégie de croissance.
\end{abstract}

Mots clés: Barcelone, tourisme, développement économique, espace public, marketing.

\title{
I. INTRODUCCIÓN
}

Desde la década de los noventa, la industria turística se ha convertido en uno de los fenómenos de transformación de la ciudad de Barcelona que mayor impacto ha tenido tanto a nivel económico como urbanístico y social. El objetivo de este artículo es analizar como el crecimiento económico de Barcelona se ha basado en la producción de una economía de servicios avanzados, en el que la visibilidad y la promoción del turismo han jugado un papel primordial. En este proceso destacan el papel promotor de macro-eventos como los Juegos Olímpicos de 1992, los cuales han sido herramientas de cambio del modelo económico de la ciudad.

La terciarización de la economía de Barcelona se hace evidente al observar la creciente importancia del sector servicios dentro de la economía local. En el año 2008, el sector servicios, que incluye el turismo, pasó a englobar el 79,2 \% de los ocupados en Barcelona. Al mismo tiempo la industria ha reducido su aportación al empleo, al ocupar el 13,8\% de los asalariados (CESB, 2009). La aportación del turismo al PIB municipal fue en el año 2008 aproximadamente del $10 \%$ del total. Se considera que el turismo generó 65.000 puestos de trabajo 
directos en Barcelona. Los turistas representaron el 65\% de las visitas a los principales museos de la ciudad, exposiciones temporales y permanentes (Turisme de Barcelona, 2010).

Este proceso de sustitución de la industria por los servicios/turismo es un rasgo común en muchas economías occidentales (Sassen, 2006). Sin embargo el caso de Barcelona merece especial atención por un grupo de factores. En primer lugar, el proceso de transformación urbanística y de imagen de la ciudad ha despertado interés en numerosas ciudades, especialmente en Europa y Latinoamérica, siendo ampliamente promovido desde la institución local y analizada por el sector público y el ámbito académico internacional. En segundo lugar, la visibilidad alcanzada por la ciudad desde los Juegos Olímpicos sitúa la experiencia de Barcelona como caso emblemático de proyección internacional a través de un macro-evento. De hecho, este es un caso excepcional, ya que a pesar de que los macro-eventos son utilizados ampliamente como mecanismos de creación de imagen y promoción económica, numerosas experiencias en otras ciudades cuestionan el nivel de éxito de la estrategia. En tercer lugar, el modelo turístico de Barcelona plantea, al mismo tiempo, un reto importante sobre el uso del espacio urbano y las características de los servicios de la ciudad, ya que incrementa el porcentaje e intensidad de los usos del suelo dedicados al consumo turístico. Ello implica la necesidad de reequilibrar tensiones que se puedan producir entre las necesidades turísticas y las de la población local. Por último, la importancia ganada por el sector turismo en Barcelona también implica un reto a la hora de buscarle alternativas o complementos en un periodo de nuevo ciclo económico.

Para dar respuesta a estas cuestiones, el presente artículo contextualiza, en primer lugar, la experiencia de Barcelona a través de una breve revisión de la literatura entorno a la importancia de la creación de imagen y visibilidad como estrategia de desarrollo urbano. A continuación se analiza cómo la celebración de los Juegos Olímpicos en 1992, la cooperación público-privada y la planificación estratégica han contribuido al desarrollo de la industria turística de la ciudad. El tercer apartado se centra en presentar el impacto económico de esta actividad y su vinculación, en cuanto a la importancia en proyección internacional, a otros sectores de nueva economía. A continuación se discute cómo la presencia turística ha influido en el uso del espacio público y los retos sociales que implica. Finalmente se concluye con una reflexión sobre el futuro de la industria en un periodo de nuevo ciclo económico.

\section{IMAGEN, VISIBILIDAD Y TURISMO COMO ESTRATEGIA DE DESARROLLO LOCAL}

El esfuerzo por crear una imagen de ciudad atractiva como estrategia de desarrollo local no es un fenómeno nuevo. Ward (1998) analiza con detalle como en el siglo XIX la estrategia de "vender un espacio" jugaba ya un papel central 
en la promoción de los nuevos enclaves de la frontera Norteamericana. Así mismo, las ciudades han gozado históricamente de atributos que las hacen atractivas como destinos turísticos. Como señala Law (1993), dada su amplia población e infraestructura, las ciudades atraen visitantes por numerosos motivos, incluyendo visitas a amigos, familiares, por razones de negocio, servicios y ocio. La fácil accesibilidad a través de las infraestructuras de transporte también les aporta una ventaja comparativa respecto a otros posibles destinos más remotos. Sin embargo, a pesar de estas ventajas estructurales, hasta los años ochenta del siglo XX pocas ciudades dedican políticas concretas a la promoción turística. Esta falta de interés en políticas específicas para el desarrollo de la industria turística se reflejaba también en el escaso interés académico, ya que hasta esta fecha los estudios académicos eran pocos y fragmentados (Edwards, Griffin y Hayllar, 2008).

Es a partir de los años ochenta cuando la creciente competencia entre ciudades para atraer inversores y actividad económica impulsa una fuerte rivalidad en la que la percepción sobre las ciudades, y más en concreto, la imagen mental que se genera sobre ellas se convierte en un componente importante del futuro éxito o fracaso económico de una urbe (Ashworth y Voogd, 1990; PallaresBarbera, 2010). Este enfoque, en el que los componentes culturales y de imagen empiezan a considerarse relevantes, aporta nuevas perspectivas sobre desarrollo local, ya que hasta esta fecha, fruto de las teorías de los polos de crecimiento de Perroux (1961), las estrategias de crecimiento económico habían girado en torno a la idea de potenciar el conjunto industrial local imbricado alrededor de una industria central y exportadora.

Inicialmente, ciudades europeas centran su nueva estrategia de promoción en el aspecto cultural. La motivación de los agentes públicos para la utilización de la cultura como estrategia de desarrollo se centra en la posibilidad de generar desarrollo económico local a la vez que se ayuda a la promoción de la ciudad. Con un cambio de imagen que produce un impacto positivo tanto en la atracción de nuevos residentes y empresas como turistas. Como ilustra el libro de Bianchini y Parkinson (1993), la regeneración económica en Europa en los años ochenta en ciudades altamente deterioradas por la desindustrialización se basa en gran medida en la utilización de la cultura como estrategia de renovación económica y urbanística. El estudio de caso de 8 ciudades medias europeas ejemplifica la interrelación entre desarrollo cultural y regeneración urbana en casos dispares, desde los antiguos centros industriales de Glasgow, Hamburgo y Bilbao, a centros académicos y regionales de Bolonia y Montpellier.

La creciente importancia de los servicios avanzados en las economías locales a partir de los años noventa incrementa la importancia de la cultura local y la imagen como herramienta de promoción, en la medida en que se considera que los trabajadores altamente cualificados prestan especial atención a cuestiones de calidad de vida en sus decisiones de localización (Krueger y Gibbs, 2007). Así, influidas por la investigación de Richard Florida (2002 y 2005), numerosas 
políticas de promoción económica de ciudades occidentales acogen la idea de clase creativa como herramienta de desarrollo.

En su esfuerzo por posicionarse internacionalmente en un contexto de globalización económica, las ciudades dedican especial atención a las políticas de promoción económica local. Como apuntan Ross y Levine (2005) este es un fenómeno que se hace muy evidente en ciudades norteamericanas. Sin embargo, esta estrategia se extiende también a Europa, donde paulatinamente las políticas urbanas acaban siendo sinónimo de política de desarrollo y crecimiento económico, trasladándose el énfasis desde las tradicionales políticas de empleo y planificación a las políticas de innovación y competitividad, tanto a nivel global (Jessop, 2002), como en el caso de Barcelona (Casellas, 2007). En esta dinámica, la industria turística se afianza como motor de la economía local.

Uno de los retos en la promoción turística de una ciudad es la consolidación de una imagen atractiva como destino turístico en un ámbito muy competitivo. Estudios sobre marketing de ciudades enfatizan que a menudo las campañas publicitarias para atraer visitantes e inversores reflejan las preferencias y gustos dominantes de los grupos de turistas, inversores o negocios que se pretende atraer, más que las características de la ciudad en concreto (Holcomb 1993, 1994). En líneas generales, temas comunes de promoción urbana se centran en las ventajas de la localización geográfica de la ciudad, su conectividad a otros centros urbanos, su dinamismo económico y cultural, su calidad de vida y medioambiente. En este contexto, la creación de imagen se hace difícil porque ciudades diversas compitiendo por el mismo tipo de clientes se promocionan con características similares (Balibrea, 2001, 2006). Un aspecto crucial para conseguir especificidad internacional es obtener singularidad propia gracias a la visibilidad de un macro-evento que "sitúe la ciudad en el mapa". En el caso de Barcelona, este punto de inflexión lo ofreció el éxito organizativo y promocional que supuso la organización de los Juegos Olímpicos de 1992.

\section{LOS JUEGOS OLÍMPICOS, LA COOPERACIÓN PÚBLICO-PRIVADA Y LA PLANIFICACIÓN ESTRATÉGICA: EJES ESTRUCTURALES DE CAMBIO}

Un breve repaso a la historia reciente de los juegos olímpicos muestra como en numerosas instancias la celebración de este macro-evento no implica el reconocimiento global de una ciudad, sino la crítica, a veces, internacional. Numerosos factores pueden enturbiar la imagen positiva que se intenta difundir. Una breve lista de ellos incluye, un diseño inadecuado de las instalaciones, una deficiente organización del evento, la retirada de patrocinadores, los costes sociales de la gentrificación producida por la demolición de viviendas y el desplazamiento de residentes, un elevado déficit público, los boicots políticos, el déficit democrático del país organizador, los ataques terroristas y los problemas de seguridad, y el posterior deterioro e inutilización de la infraestructura depor- 
tiva, entre otros (Tomlinson, 1999; Lenskyj, 2002; Preuss, 2004; Payne, 2005). La amplia difusión de los medios de comunicación de la represión política en China, de las dificultades de Atenas para finalizar su infraestructura olímpica, o de la mala organización del evento en Atlanta ${ }^{2}$ ejemplifican los retos de promoción que suponen los juegos.

Sin embargo, y a pesar de las incertidumbres que el evento genera, los juegos se perciben como una oportunidad única de impulso de la ciudad. El caso de Barcelona puede interpretarse como un ejemplo paradigmático de éxito en marketing de ciudad. Al mismo tiempo la celebración del evento muestra la posibilidad de regeneración urbanística que los Juegos implican, en la medida en que más del $80 \%$ del total de inversión se dedicó, en el caso de Barcelona, a proyectos urbanos e infraestructura no ligada a espacios deportivos (Varley, 1992; Brunet, 1995; Gold y Gold, 2008).

A nivel de gobernanza local, los Juegos en Barcelona ofrecen la primera clara necesidad de colaboración público-privada en la ciudad (Raventós, 2000). La organización del evento permite al sector público diseñar unos objetivos de transformación urbanística a gran escala, pero para ello la administración necesita la inversión del sector privado. A su vez, el sector privado reconoce la posibilidad de asegurarse las inversiones a través de su participación activa en los proyectos. El reconocimiento de la mutua necesidad entre agentes del sector público y privado en Barcelona abre la posibilidad de cooperación y reciprocidad mutua en la ciudad. Esta cooperación se afianzará en los años siguientes como consecuencia del éxito de los Juegos y la planificación estratégica (Casellas, 2006).

La planificación estratégica ha sido una de las medidas en política local más difundidas, y que mayor interés ha generado, bajo el concepto del "Modelo Barcelona". El primer Plan Económico y Social se inicia a mediados de los años ochenta, en el contexto de la reforma urbanística entorno a la organización de los Juegos Olímpicos. Como ya se ha apuntado, los Juegos suponen el pretexto para ejecutar en un corto plazo de tiempo una gran reforma urbana a escala de ciudad (Maragall, 1999). Para ello era importante planificar de forma coordinada. El primer plan estratégico se aprobó en 1990. A éste le siguieron el segundo plan, aprobado en 1994, y el tercero, aprobado en 1999. Es importante destacar que, a pesar de que la planificación estratégica en Barcelona se ha planteado como ejemplo participativo dada la presencia de amplias capas de la sociedad, de hecho las comisiones, aunque amplias en el número de participantes y sec-

2 En 1992, al finalizar los juegos en Barcelona, el comité organizador de los juegos en Atlanta, ACOG en su acrónimo en inglés, publicitaba que se comprometía a organizar los mejores juegos olímpicos de la historia. Para ello Atlanta mejoraba sus sistemas de infraestructuras de transporte y hotelero, mientras garantizaba la total seguridad durante la celebración. Sin embargo, poco después de la inauguración del evento, los medios de comunicación internacionales y nacionales ironizaron ampliamente sobre el fracaso de la organización, y el acrónimo ACOG pasó a ser identificado como: "Atlanta cannot organize the games". 
tores implicados, han estado compuestas por técnicos y especialistas. Los planes se hicieron públicos a la ciudadanía a través de conferencias y seminarios, en los que los asistentes pudieron hacer contribuciones con críticas y sugerencias (Marshall, 1990; Casellas, 2003).

El éxito de la planificación, en la medida en que ha permitido generar un proceso de diálogo entre intereses de la administración y los agentes económicos, y la necesidad de considerar un espacio económico y social funcional más amplio que el estrictamente delimitado por el municipio, conducen a la aprobación en el 2003 del Primer Plan Estratégico Metropolitano de Barcelona (PEMB), el cual integra 36 municipios que conforman el área metropolitana. Tanto en los tres anteriores planes locales, como en el plan metropolitano, se hace especial énfasis en la importancia de la cooperación público-privada.

Fruto de la importancia de la cooperación entre el sector público y privado se generan en Barcelona a partir de principios de los noventa numerosos instrumentos de promoción internacional de la ciudad. Estos instrumentos son de carácter sectorial, ya que se basan en el fomento específico de determinados sectores económicos - digital, universitario, logístico, biomédico, y turístico, como los más significativos. Todos ellos son mixtos, porque aúnan los esfuerzos y recursos del sector público y privado.

Para capitalizar la promoción de la imagen generada por los Juegos, en 1992 el Ayuntamiento, la Cámara de Comercio Industria y Navegación de Barcelona, y la agencia privada de promoción Barcelona Promoción comisionan un plan estratégico de turismo para la ciudad. El Primer Plan Estratégico de Turismo de Barcelona es aprobado en 1993. La comisión de asesores del plan implicó a treinta expertos representantes de diecinueve sectores, incluyendo congresosferias, deportes, diseño, cultura y universidades. La comisión concluyó que la ciudad tenía un alto potencial para convertirse en un destino turístico atractivo si los recursos presentes eran maximizados y si nuevos productos eran desarrollados en un futuro inmediato (Pla Estratègic de Turisme, 1994). Los objetivos del primer plan estratégico de turismo incluirán la propuesta de los rasgos de organización y financiación de una agencia para ejecutar las estrategias de promoción. Siguiendo estas recomendaciones, la nueva agencia público-privada, Consorci de Turisme de Barcelona (CTB), entra en vigor en enero de 1994.

El CTB está organizado en un consejo general presidido por el alcalde de la ciudad; un comité ejecutivo que integra vocales del Ayuntamiento, Cámara de Comercio de la ciudad y la fundación privada de promoción turística; y un director general. El director administrativo de CTB a finales de los años noventa, Ignasi de Delàs (1999), indicaba que los objetivos iniciales del consorcio incluían la consolidación del turismo existente, sobre todo el turismo que se vinculaba a congresos y ferias, y la atracción de nuevo turismo, con especial énfasis en la promoción de Barcelona como un destino vacacional. Con el fin de promover Barcelona a nivel nacional e internacional como destino turístico, el CTB desarrolló en los años noventa seis programas, los cuales incluían los sectores de comercio, convenciones, cultura y ocio, compras, atención al turismo y desarro- 
llo del producto turístico. Alfonso Escolà (1999), miembro del departamento de comercio del Ayuntamiento, aseguraba que para el período 1994-1998, en el cual ya se podía valorar los resultados del CTB en la promoción de Barcelona, la agencia había producido unos resultados excelentes.

Como empresa mixta, CTB combina la financiación de recursos públicos con los privados generados desde el propio consorcio a través de la promoción de productos turísticos (bus turístico, walking tours que ofrecen rutas guiadas a pie, Barcelona card como tarjeta de descuentos para visitantes, entre otros). La financiación pública se ha mantenido en aproximadamente 2 millones de euros aportados por partes iguales entre el Ayuntamiento y la Cámara de Comercio, desde la creación de la entidad. En el año 2008, con un presupuesto de 34,6 millones de Euros, la financiación generada desde la propia entidad representaba más del $90 \%$ del total. El elevado porcentaje de recursos propios permite al Consorcio presentarse como una entidad independiente que "se rige prioritariamente por criterios técnicos" (Consorci Turisme de Barcelona, 2009b: 7).

\section{EL IMPACTO ECONÓMICO DEL TURISMO Y EL NUEVO MODELO DE CRECIMIENTO URBANO: BARCELONA "ON THE MAP"}

A pesar de la importancia creciente del sector turismo para la ciudad, y siguiendo una endémica falta de datos económicos desagregados a escala local en comparación a países anglosajones, en España no existe un sistema estadístico sistemático de recopilación de datos fiables sobre el impacto económico del turismo a escala local, en términos de contribución al PNB, salarios y puestos de trabajo. Estimaciones realizadas por CTB apuntan que, en el año 2008, el turismo ocupó entre 80.000 y 100.000 personas y generó entre 7.000 y 8.000 millones de euros. Con un gasto de 20 millones de euros, la contribución de la industria turística a la ciudad se había doblado respecto al 2001. El número total de visitantes, tomando en cuenta los 6,6 millones que se alojaban en hoteles y otros establecimientos turísticos, y aquellos que lo hacían en casa de familiares o amigos, o no pernoctaban en la ciudad, situaban el número total de visitantes en 18 millones.

Estos datos, aun con las limitaciones que implica para su análisis la falta de series temporales y los problemas metodológicos en su recopilación, permiten identificar la importancia del turismo como sector económico de la ciudad. De hecho, en el año 2005 la consolidación de Barcelona como destino turístico se consideraba alcanzada y el turismo se afianzaba como un sector estratégico. Joan Clos, alcalde de Barcelona en este periodo, afirmaba que los rasgos que caracterizan Barcelona como ciudad atractiva eran: el carácter emprendedor, el impulso decidido por la innovación, y la larga tradición comercial y de servicios (Ajuntament de Barcelona, 2005). Ello le llevaba a afirmar que Barcelona se situaba entre las ciudades más avanzadas de Europa. Los objetivos de futuro en cuanto a promoción turística incluían atraer visitantes en calidad de turistas o 
consumidores de ocio o cultura. Entre las propuestas con más futuro para la industria turística se reconocían la proliferación y éxito de las compañías aéreas de bajo coste, el aumento del parque hotelero de la ciudad, la fortaleza creciente del turismo de reuniones, y el éxito de las tematizaciones como fórmula de promoción - Año Internacional Gaudí 2002; Año del Deporte 2003; Fórum Barcelona 2004; Año de la Alimentación, Cocina y Gastronomía 2005-2006; Picasso Barcelona, 2006.

A finales de la década de los años 2000, el creciente número de visitantes y pernoctaciones desde el periodo preolímpico reafirmaba el éxito de Barcelona como destino turístico. El año record para el periodo 1990-2008 se situaba en el 2007, según datos de CTB (2009a). Ello comportaba un nivel de crecimiento turístico y proyección internacional que situaba Barcelona en el ranking de ciudades más visitadas de Europa (cuadro I).

Cuadro I - Ranking de las principales ciudades turísticas europeas (Número de turistas).

Table I - Ranking of principal European tourist destinations (Number of tourists).

\begin{tabular}{l|lrrc} 
& \multicolumn{1}{|c}{ Ciudad } & $\mathbf{2 0 0 6}$ & $\mathbf{2 0 0 7}$ & $\begin{array}{c}\text { Variación \% } \\
\mathbf{2 0 0 7} / \mathbf{2 0 0 6}\end{array}$ \\
\hline $\mathbf{1}$ & Londres (Gran) & 26600000 & 25450000 & $-4,32$ \\
\hline $\mathbf{2}$ & París & 16379938 & 15399817 & $-5,98$ \\
$\mathbf{3}$ & Roma & 11158447 & 11607000 & 4,68 \\
$\mathbf{4}$ & Madrid & 8645572 & 9307723 & 7,66 \\
$\mathbf{5}$ & Barcelona & 7236428 & 7689394 & 6,26 \\
$\mathbf{6}$ & Berlín & 7070239 & 7585283 & 7,28 \\
$\mathbf{7}$ & Dublín & 5676000 & 5765000 & 1,57 \\
$\mathbf{8}$ & Ámsterdam & 4659600 & 4863600 & 4,38 \\
$\mathbf{9}$ & Munich & 4371322 & 4701717 & 7,56 \\
$\mathbf{1 0}$ & Viena & 4377449 & 4636302 & 5,91 \\
$\mathbf{1 1}$ & Praga & 4142538 & 4485372 & 8,28 \\
\hline
\end{tabular}

Fuente: Consorci Turisme de Barcelona (2009a).

Tomado como indicador el número de turistas en hoteles según nacionalidad (cuadro II), observamos la creciente internacionalización del sector para el periodo 1990-2008, con una presencia muy significativa del turismo de origen europeo. La estrategia promocional futura pasa, por tanto, por incrementar la atracción de la ciudad como destino turístico de viajeros procedentes de América del Norte, y abrirse tanto a países emergentes como China, India, Brasil y Rusia con una creciente población con poder adquisitivo; como a nuevos grupos demográficos, de los que destacan la tercera edad y los prejubilados. 
Cuadro II - Número de turistas en hoteles según nacionalidad (\%).

Table II - Number of tourists in hotels by nationality (\%).

\begin{tabular}{l|ccccccc}
\multicolumn{1}{c}{} & $\mathbf{1 9 9 0}$ & $\mathbf{1 9 9 2}$ & $\mathbf{1 9 9 4}$ & $\mathbf{1 9 9 6}$ & $\mathbf{1 9 9 8}$ & $\mathbf{2 0 0 6}$ & $\mathbf{2 0 0 8}$ \\
\hline \multicolumn{1}{l}{ España } & 51,2 & 43,7 & 38,5 & 36,9 & 37,8 & 30,0 & 29,2 \\
\hline Europa & -- & 39,0 & 44,1 & 41,4 & 45,8 & 51,0 & 50,9 \\
\hline \multicolumn{1}{c}{ Francia } & 7,3 & 8,8 & 11,0 & 8,7 & 11,1 & 7,0 & 6,8 \\
$\quad$ Italia & 6,1 & 9,6 & 7,0 & 5,4 & 9,0 & 9,0 & 8,2 \\
$\quad$ Reino Unido e Irlanda & 4,1 & 6,8 & 7,0 & 8,8 & 8,8 & 13,0 & 11,5 \\
$\quad$ Alemania & 3,4 & 7,0 & 7,0 & 5,1 & 6,7 & 5,0 & 5,2 \\
$\quad$ Resto de Europa & -- & 6,8 & 13,0 & 13,4 & 10,2 & 17,0 & 19,2 \\
\hline \multirow{2}{*}{ EEUU/Canadá } & 5,9 & 5,0 & 8,9 & 9,0 & 8,1 & $7,0 *$ & $7,0 *$ \\
Resto de América & 3,9 & 5,8 & 4,6 & 3,8 & 3,2 & 4,0 & 4,7 \\
Japón & 2,6 & 4,5 & 2,1 & 2,2 & 1,6 & 2,0 & 2,0 \\
Resto del mundo & -- & 2,0 & 1,8 & 6,7 & 3,5 & 6,0 & 6,2 \\
\hline
\end{tabular}

*Sólo incluye EEUU.

Fuente: Consorci Turisme de Barcelona (2009a).

Respecto a los objetivos propuestos a mediados de los noventa, centrados en la consolidación del turismo existente vinculado a congresos y ferias, y a la atracción de nuevo turismo vacacional, los datos estadísticos muestran la consecución de estas metas diez años después. El turismo por razones profesionales aumentó a lo largo de este periodo en términos absolutos, pero respecto al total, el porcentaje disminuyó significativamente, pasando de cerca del 70\% en 1990, a cerca del $51 \%$ en el año 2006 (cuadro III).

Cuadro III - Turistas según motivo del viaje, 1990-2008 (\%).

Table III - Purpose of the visits, 1990-2008 (\%).

\begin{tabular}{lccccccc} 
& $\mathbf{1 9 9 0}$ & $\mathbf{1 9 9 4}$ & $\mathbf{1 9 9 6}$ & $\mathbf{1 9 9 8}$ & $\mathbf{2 0 0 0}$ & $\mathbf{2 0 0 6}$ & $\mathbf{2 0 0 8}$ \\
\hline Profesional & 69,1 & 57,9 & 54,9 & 47,3 & 45,7 & 50,4 & 48,4 \\
Vacacional & 22,7 & 31,3 & 36,4 & 51,8 & 51,5 & 46,4 & 48,1 \\
Personal y otros & 8,2 & 10,8 & 8,7 & 0,9 & 2,8 & 3,2 & 3,5 \\
\hline & 100 & 100 & 100 & 100 & 100 & 100 & 100 \\
\hline Total turistas & 1732902 & 2663887 & 3061994 & 2969490 & 3141162 & 6709175 & 6659075 \\
\hline
\end{tabular}

Fuente: Consorci Turisme de Barcelona (2009a).

Este cambio de tendencia ha sido consecuencia del aumento espectacular del turismo vacacional, que ha ganado importancia a partir de finales de los noventa. Con respecto a la variable género, el porcentaje de hombres visitantes 
respecto al de las mujeres también ha decrecido para este periodo, y si bien el número de visitantes masculinos es todavía muy superior al de mujeres, la diferencia porcentual entre ambos sexos ha disminuido (cuadro IV). Estas variables permiten argumentar que el turismo vacacional y familiar se ha ido afianzando y ha ido ganando importancia, en el cómputo total de visitantes.

Cuadro IV- Turistas según género, 1990-2008 (\%).

Table IV-Tourists by gender, 1990-2008 (\%).

\begin{tabular}{lccccccc} 
& $\mathbf{1 9 9 0}$ & $\mathbf{1 9 9 2}$ & $\mathbf{1 9 9 4}$ & $\mathbf{1 9 9 6}$ & $\mathbf{2 0 0 0}$ & $\mathbf{2 0 0 6}$ & $\mathbf{2 0 0 8}$ \\
\hline Hombre & 79,8 & 66,4 & 65,9 & 65,8 & 59,5 & 63,1 & 60,2 \\
Mujer & 20,2 & 33,6 & 34,1 & 34,2 & 40,5 & 36,9 & 39,8 \\
\hline & 100 & 100 & 100 & 100 & 100 & 100 & 100 \\
\hline
\end{tabular}

Fuente: Consorci Turisme de Barcelona (2009a).

A nivel de promoción turística, a finales de la década del 2000, el éxito de la industria turística en Barcelona se identifica como resultado de la acción del consorcio público-privado CTB, y la combinación de recursos turísticos diversos, incluyendo la segmentación de la oferta turística en los sectores culturales, de ocio y entretenimiento, compras, cruceros, congresos, convenciones y viajes de incentivos. Entre los segmentos de mayor éxito se sitúa el de congresos (fig. 1). Desde CTB se enfatizaba que el creciente número de visitantes ligados a congresos y ferias es el resultado del atractivo de la ciudad, la creciente oferta de espacio y calidad de los centros de convenciones, las características del parque hotelero y las estrategias de promoción del sector. Una de las estrategias clave de la ciudad en cuanto a congresos ha sido el optar por el mercado internacional, desmarcándose de la competencia generada a nivel nacional. Otro segmento con un gran crecimiento es el ligado a cruceros, en el que Barcelona se consolida como el mayor puerto en el Mediterráneo.

El escenario de crecimiento de la industria turística, con repercusiones económicas positivas para la ciudad, ha tenido sin embargo implicaciones cuestionables a nivel de uso del espacio público. Los conflictos empezaron a manifestarse de forma acuciante a finales de la década de 2000. Respondiendo a las críticas de ciertos sectores sociales, especialmente la prensa y representantes de colectivos residentes en el centro urbano, en el año 2008 y bajo el liderazgo del Ayuntamiento, el CTB inicia un nuevo proceso de reflexión para la elaboración de un nuevo plan estratégico de turismo. De forma significativa, bajo el nombre "Plan Estratégico de Turismo 2015 de la ciudad de Barcelona" el nuevo plan introduce el componente ciudad, y por extensión, ciudadanía en su ámbito de análisis.

Las implicaciones de un mayor uso del espacio urbano, tanto a nivel cualitativo como cuantitativo y las respuestas del nuevo plan son analizados en el siguiente apartado. 


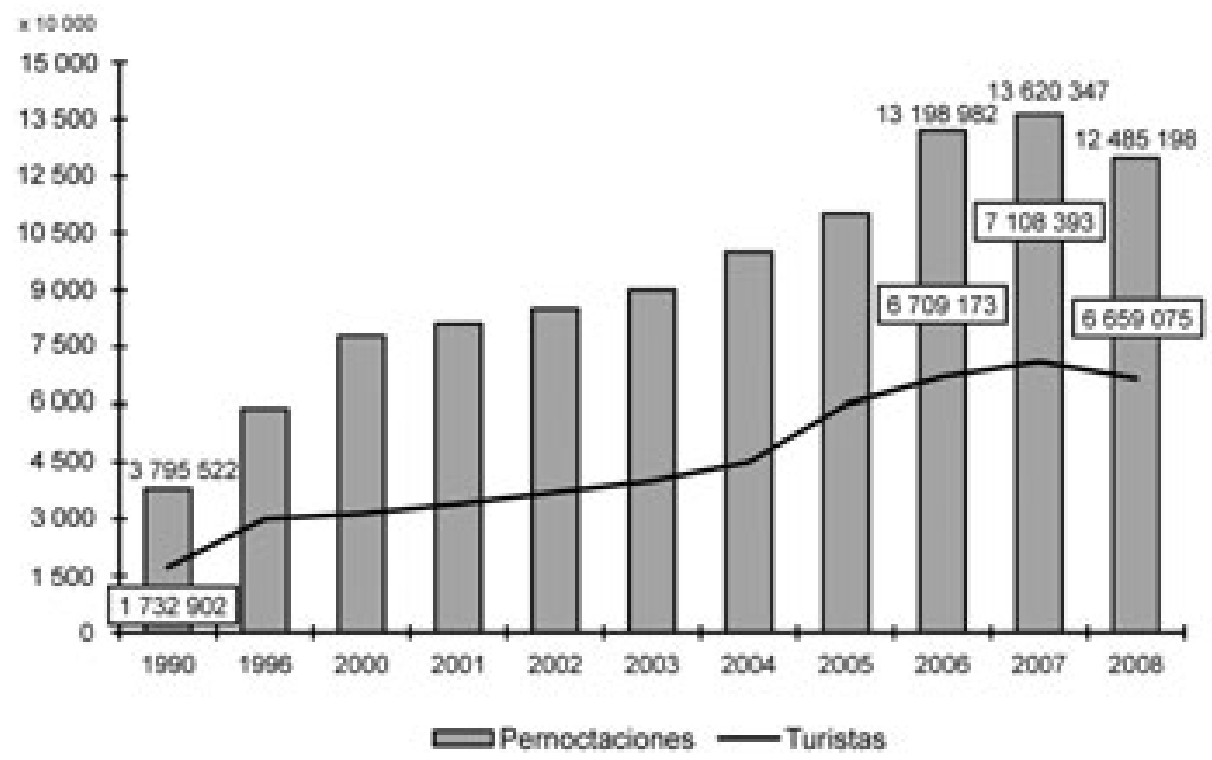

Fuente: Consorci Turisme de Barcelona (2009a)

Fig. 1 -Turistas y pernoctaciones en hoteles (1990-2008).

Fig. 1 - Number of hotel guests and overnight stays (1990-2008).

\section{EL TURISMO Y EL ESPACIO URBANO Y SOCIAL: CONSIDERACIONES, OPORTUNIDADES Y RETOS}

El creciente énfasis en la promoción de la industria turística en ciudades influye en el uso del espacio urbano en la medida en que se acrecienta significativamente el porcentaje de espacio público y productivo destinado al consumo y la producción ligada al turístico. Centros históricos, marinas, parques, monumentos, edificios singulares e infinidad de museos se convierten en espacios preferentemente turísticos (Gospodini, 2001). En el caso de Barcelona, la ciudad revaloriza una amplia gama de su patrimonio urbano como producto turístico, a través de una relectura del patrimonio monumental y urbanístico generado por procesos políticos, sociales y económicos contradictorios a lo largo de su historia (Casellas, 2009). En este proceso, el centro histórico, o Barrio Gótico de Barcelona, con su conjunto de arquitectura religiosa y civil "reinventada" a mediados del siglo diecinueve (Ganau, 2008), se convierte a principios del siglo veintiuno en una de las áreas más visitadas de la ciudad.

Como sucede en otras ciudades occidentales, a partir de la década de los años noventa desde las políticas urbanas se genera en Barcelona una mayor atención hacia lo cultural y simbólico como herramienta de crecimiento econó- 
mico. Esta dinámica, definida como economía simbólica por Zukin (1992) implica el reconocimiento de la capacidad del capital no-material o simbólico de influir directamente en el desarrollo local a través de los incentivos que aporta al mercado laboral, inmobiliario, financiero o turístico, entre otros. Ello comporta una nueva concepción del espacio urbano en la que el valor de cambio del espacio, su valor monetario, se incrementa y, eventualmente, impera sobre su valor de uso. Como consecuencia de esta nueva tendencia, el sector público en sus decisiones de inversión prioriza determinadas infraestructuras o proyectos que añaden visibilidad e imagen a la ciudad. En el caso de Barcelona, la cultura se convierte en uno de los sectores más competitivos (Balibrea, 2006). En la medida en que los recursos públicos son limitados, ello resulta en un detrimento de otras posibles políticas de carácter más social, lo cual ha generado las críticas de diversos colectivos a partir de finales de los años noventa.

Al interés del sector público en promover políticas de desarrollo económico por encima de las tradicionales políticas sociales y laborales, se añade la presión ejercida por el turismo sobre el espacio urbano del centro histórico de la ciudad. La presencia masiva de turismo urbano concentrado en centros históricos es común en ciudades que cuentan con un reconocido patrimonio artístico, cultural y urbano como Venecia, Roma o París. Sin embargo, en el caso de Barcelona, el polo de atracción turística que genera el centro antiguo es relativamente reciente y es el resultado de la combinación de una serie de factores urbanísticos y promocionales.

El distrito de Ciutat Vella es un área urbana central que, similar a otros centros urbanos en España, sufrió un grave deterioro durante el periodo de la Dictadura Franquista (1939-1975). En Barcelona, a la desinversión general a nivel español en infraestructuras, servicios urbanos y mantenimiento del parque de viviendas de este periodo, se debe añadir el deterioro causado por la alta densidad poblacional del centro, la elevada concentración de población inmigrante sin recursos económicos, el envejecimiento de la población y la grave problemática social y económica causada por la crisis de los años setenta (Ràfols, 1997; Fabre y Huertas, 2000). Dado el patrimonio urbanístico y simbólico de este centro, tanto para Barcelona como para Cataluña y la amplia presencia institucional y política en la zona, desde principios de los años ochenta y siguiendo una estrategia de regeneración de espacios públicos por parte del Ayuntamiento (Bohigas, 1985; Capel, 2007) se inició una intervención a gran escala dirigida por el sector público, quién aplicó mecanismos especiales de gestión para la rehabilitación integral de la zona.

Los mecanismos de gestión auspiciados desde el Ayuntamiento, y basados en legislación urbanística aprobada por el gobierno central, incluyeron la cooperación interinstitucional entre administraciones a través de la creación de Áreas de Rehabilitación Integrada (ARIs) en 1986; la creación de una Comisión Gestora del ARI con participación de las asociaciones vecinales en 1987; el diseño de proyectos de reforma urbanística a través de Planes Especiales de Reforma Interior (PERIs) para diferentes sectores; y la creación en el año 1988 de una 
agencia público-privada encargada de todo el proceso de regeneración urbanística, Promoció Ciutat Vella S.A., sustituida el año 2000 por la agencia Foment de Ciutat Vella S.A.

Una de las actuaciones más importantes de esta regeneración urbana se realizó en el barrio de El Raval, colindante al Barrio Gótico, en el centro urbano de la ciudad. Este barrio era el espacio más degradado urbanística y socialmente en la ciudad a principios de los años ochenta. Su renovación se diseñó capitalizando en el escaso patrimonio urbanístico y cultural de la zona norte del barrio. Siguiendo esta estrategia, el primer plan diseñado por el Ayuntamiento a principios de los ochenta se materializa con la creación de un cluster cultural, que incluye un centro cultural y de investigación sobre temas urbanos - Centre de Cultura Contemporánea de Barcelona (CCCB) inaugurado en 1994, y el Museo de Arte Contemporáneo de Barcelona (MACBA) diseñado por Richard Meier y finalizado en 1995. A este grupo cultural se le añaden centros universitarios y de investigación a finales de la década. Finalmente, la transformación morfológica más radical, bajo el denominado Plan Central del Raval, tiene lugar en los noventa con la demolición de 125 bloques de viviendas que afectaron a 1.384 apartamentos y 293 negocios en la zona más cercana al puerto. La reforma permitió la creación de una nueva avenida y nuevos bloques de viviendas y negocios, incluyendo la construcción de un hotel ampliamente protestado por grupos de residentes que se oponían al impacto visual del edificio y sus implicaciones sobre el tejido social y económico del barrio (PROCIVESA, 2002; Casellas, 2003).

Como el caso del Pla Central del Raval ejemplifica, los procesos de transformación urbana en el centro histórico han ido acompañados de una revalorización del suelo, el cual ha supuesto un paulatino proceso de gentrificación, o desplazamiento de antiguos residentes con pocos recursos económicos por nuevas capas sociales, especialmente parejas jóvenes sin hijos que valoran la posición central del barrio y su creciente oferta cultural y de ocio. El fenómeno de la gentrificación afectó inicialmente a calles y espacios centrales del distrito de Ciutat Vella a finales de los años noventa, y se extendió hacia gran parte del tejido urbano de la zona durante la década del 2000. En la medida en que la ejecución de numerosos planes de restructuración urbana dirigidos desde el sector público esponjó el territorio, demolió manzanas de viejos edificios y creó nuevos espacios orientados al ocio y el turismo la gentrificación del barrio creció. Sin embargo, el fenómeno iniciado en la década de los noventa y que se auguraba muy intenso (Martínez, 2001), se vio mitigado como consecuencia de la creciente presencia de población inmigrante a partir de finales de los noventa, fruto del modelo de crecimiento económico español basado en creación de puestos de trabajo no cualificados en construcción y servicios. Esta población inmigrante se concentró en gran medida en el centro urbano (Pareja y Tapada, 2000) en un periodo en el que, a su vez, se acrecentó la presencia del turismo y los servicios orientados a éste.

Los datos de visitantes a principales centros de interés revelan que la ciudad en general, y especialmente ciertos edificios y museos emblemáticos del centro 
se convirtieron en productos turísticos de gran atractivo (cuadro V). Otro polo de atracción turística del centro lo forma el frente litoral, que empezó a recuperarse a mediados de los años ochenta, y que sufrió una transformación radical en el contexto de los Juegos Olímpicos de 1992, con la creación de nuevas marinas, playas y barrios residenciales colindantes al tradicional barrio obrero de La Barceloneta (Bohigas, Buchanan, Magnano Lampugnani, 1991; Martorell, Bohigas, Mackay, Puigdomenech, 1992; Mackay, 2000).

Cuadro V - Número de visitantes a los lugares de interés (1994-2008).

Table V-Visitor numbers of places of interest (1994-2008).

\begin{tabular}{|c|c|c|c|c|c|c|c|}
\hline & 1994 & 1996 & 1998 & 2000 & 2006 & 2008 & $\begin{array}{c}\text { Variación \% } \\
2008 \text { / } 1994\end{array}$ \\
\hline \multicolumn{8}{|l|}{ Museos y Centros Culturales } \\
\hline Sagrada Familia & 700000 & 874865 & 1094015 & 1420087 & 2542070 & 2731690 & 390 \\
\hline Museo Picasso & 711103 & 822674 & 1000368 & 1026549 & 1234454 & 1330171 & 187 \\
\hline Fundación Joan Miró & 236196 & 330232 & 439981 & 497295 & 1016299 & 1070692 & 453 \\
\hline $\mathrm{CCCB}$ & 250394 & 373208 & 323523 & 378145 & 351426 & 377567 & 150 \\
\hline CosmoCaixa Barcelona & 419709 & 408706 & 255628 & 262561 & 2008312 & 2608985 & 621 \\
\hline Museo de Historia de la Ciudad & 168876 & 229921 & 143490 & 172421 & 389028 & 554501 & 328 \\
\hline MACBA & -- & 240059 & 289035 & 173098 & 474315 & 660200 & $275^{*}$ \\
\hline \multicolumn{8}{|l|}{ Equipamientos de Ocio } \\
\hline Aquarium & -- & 1678000 & 1557185 & 1563493 & 1443847 & 1611259 & $-4^{*}$ \\
\hline Montjuic Poble Espanyol & 1421396 & 1361349 & 1355296 & 1478546 & 1337596 & 1321020 & $-8^{*}$ \\
\hline $\operatorname{Imax}$ & -- & 929052 & 1124208 & 818707 & 448948 & 440540 & $-53 *$ \\
\hline Parque del Laberinto & -- & 640142 & 142000 & 127129 & 146940 & 156265 & $-76^{*}$ \\
\hline
\end{tabular}

*La variación porcentual se calcula respecto el año 1996

Fuente: Consorci Turisme de Barcelona (2009a).

La gran concentración de turismo en el centro de la ciudad ha generado críticas entre grupos de población local que sufren alguna de las externalidades negativas que la actividad genera (polución acústica, masificación de infraestructuras de transporte y espacios públicos, etc.). La fase de diagnóstico del nuevo plan estratégico de turismo identificaba como problemas más acuciantes en el 2010, la congestión de espacios públicos, los problemas de movilidad, la falta de control y regulación de numerosos apartamentos turísticos que proliferaron en el centro histórico, y la falta de profesionalización de la industria que ofrecía bajos salarios y contratos precarios a muchos de los trabajadores del sector (Pla Estratègic de Turisme de la Ciutat de Barcelona, 2008).

Junto a la necesidad percibida por los agentes promotores de la actividad turística de mitigar la tensión surgida entre los ciudadanos y los turistas en la primera década del 2000 - bautizada en algunos medios de comunicación como "turismofobia" -, se unía la obligación de mantener y mejorar, en lo posible, la opinión que los turistas tenían de Barcelona. En líneas generales, la ciudad 
gozaba de buena reputación entre sus visitantes (cuadro VI). Entre los aspectos más valorados se encontraba precisamente la ciudad en sí misma, es decir, las características urbanísticas y arquitectónicas, junto a la oferta cultural y de ocio. Entre los puntos más débiles se haya elementos de polución vinculados a la estructura de ciudad compacta y el modo de vida mediterráneo, destacando la contaminación atmosférica y los ruidos.

Cuadro VI - Opinión sobre diferentes aspectos de Barcelona, 1990-2008 (escala del 1 al 10).

Table VI - Survey on different aspects of Barcelona, 1990-2008 (on a scale of 1 to 10).

\begin{tabular}{lccccc} 
& $\mathbf{1 9 9 0}$ & $\mathbf{1 9 9 2}$ & $\mathbf{1 9 9 6}$ & $\mathbf{2 0 0 6}$ & $\mathbf{2 0 0 8}$ \\
\hline Oferta arquitectónica & 8,0 & 8,3 & 8,1 & 8,5 & 8,6 \\
Oferta Cultural & 7,8 & 7,7 & 7,7 & 8,2 & 8,3 \\
Ocio y entretenimiento & 7,3 & 7,4 & 8,0 & 8,0 & 8,0 \\
Carácter de los ciudadanos & 7,7 & 7,4 & 7,2 & 8,0 & 7,6 \\
Transporte Público & 7,4 & 7,1 & 7,7 & 8,1 & 7,8 \\
Infraestructuras & 6,8 & 6,3 & 7,9 & -- & 7,9 \\
Señalización e información & 6,7 & 6,5 & 6,7 & 7,8 & 7,9 \\
Seguridad & 6,3 & 6,2 & 5,8 & 7,2 & 7,1 \\
\hline Relación calidad/precio: & & & & & \\
\hline \multicolumn{1}{c}{ Restaurantes } & 6,7 & 6,7 & 7,3 & 7,6 & 7,7 \\
$\quad$ Comercio & 6,8 & 5,7 & 7,3 & 7,8 & 7,7 \\
$\quad$ Hoteles & 6,2 & 6,4 & 7,3 & 7,3 & 7,6 \\
\hline Contaminación: & & & & & \\
\hline \multicolumn{1}{c}{ Atmosférica } & -- & 4,9 & 5,0 & 6,5 & 6,8 \\
$\quad$ Ruidos & -- & 4,8 & 5,0 & 6,3 & 6,4 \\
\hline Limpieza general & -- & 5,4 & 5,0 & 7,4 & 7,2 \\
\hline Valoración global & -- & -- & 7,8 & 8,2 & 7,9 \\
\hline$\quad$
\end{tabular}

Fuente: Consorci Turisme de Barcelona (2009a).

\section{REFLEXIONES FINALES ENTORNO A LA "MARCA BARCELONA"}

Aunque a diferencia de numerosas ciudades occidentales, Barcelona no ha realizado nunca campañas promocionales importantes en soportes publicitarios internacionales publicitándose como ciudad, de hecho, a través de su estrategia de promoción sectorial, Barcelona ha conseguido crear, tal y como identifican los agentes económicos y políticos locales, la Marca Barcelona (Homs, 2010). Esta marca viene asociada a una imagen internacional de ciudad innovadora, atractiva, con calidad de vida y con amplio interés para diversos segmentos económicos y turísticos (Ajuntament de Barcelona, 2005). La internacionalización de su turismo a finales de la década del 2000 permite afianzar la importancia económica del sector, tanto para Barcelona como para España, ya que con el $70 \%$ de turismo de origen extranjero, la industria actúa como sector exportador. 
El éxito de las estrategias de la industria turística a corto-medio plazo en Barcelona a principios de la década del 2010 depende tanto de factores exógenos como endógenos a la propia ciudad. Como factores exógenos se perfilan como importantes la evolución de la crisis económica iniciada en el 2007 en países desarrollados y emergentes de los cuales provienen los turistas; y las limitaciones que implican los posibles futuros retos energéticos, especialmente la posibilidad de un incremento del precio del petróleo con su efecto negativo en empresas de transporte tanto aéreo como marítimo. De entre los factores endógenos cabe destacar las tensiones que se generan entre las necesidades turísticas y las de la población local. Por un lado, un modelo de ciudad orientado al crecimiento del sector turismo puede comportar, entre otros, la necesidad de ampliación del aeropuerto y la creación de hubs aéreos; al igual que una mayor presencia de infraestructuras y servicios turísticos. A estas necesidades se debe contraponer la capacidad de decisión de la clase política catalana y de la ciudad, con un poder limitado sobre decisiones de inversión y gestión de infraestructuras como el aeropuerto; pero, también y de forma muy especial, de la voluntad de la población local de aceptar una creciente presencia de turistas y de infraestructuras, y servicios asociados a ellos.

Sobre este último punto, y con el objetivo de mitigar las externalidades negativas sobre áreas turísticas muy congestionadas como son el centro urbano y algunos monumentos específicos vinculados al modernismo y Gaudí, el nuevo Plan Estratégico de Turismo 2015, se plantea la necesidad de establecer mayores límites territoriales a la Marca Barcelona y una redistribución de destinos dentro de la ciudad (Ajuntament de Barcelona, 2005; Homs, 2010). Para ello se trabaja con el objetivo de identificar amenidades ubicadas en barrios periféricos, y en poblaciones y espacios de la región metropolitana, e incluso a escala catalana. Así mismo, se apunta a la necesidad de mejorar la situación laboral del sector, actuando para fomentar un marco normativo que ampare la calidad en la contratación y las relaciones laborales. Finalmente, se hace especial énfasis en la necesidad de crear una conciencia positiva en el ciudadano sobre la aportación del sector turístico a la ciudad. El éxito de estas medidas dependerá de la capacidad de la ciudad de reestructurar el sector turístico, pero también, de la dinámica de la económica internacional.

Otro aspecto que preocupa, con respecto a la seguridad de los turistas, y que no ha sido tratado abiertamente, dado los efectos negativos que producen a la imagen positiva, es lo que eufemísticamente se tipifica de "pequeños hurtos". Un breve repaso a la red permite encontrar numerosas páginas de visitantes y entidades públicas y privadas aportando recomendaciones sobre como protegerse de los numerosos hurtos que los turistas sufren especialmente en el casco antiguo, las estaciones de trenes y autobuses, las playas y el metro. Este es un problema que no se ha afrontado abiertamente y que genera un gran malestar entre las victimas, cuyo número es muy difícil de cuantificar, porque en el caso de que no se pierdan documentos importantes, muy a menudo, se opta por no denunciar el hurto. 
A principios de la década del 2010, la ciudad de Barcelona afronta el reto de mantener su nivel de éxito promocional ante los dilemas generados por la posible saturación del sector turístico, y la incertidumbre entorno a la capacidad de la economía española e internacional de superar la crisis económica a corto plazo. El futuro de la economía en Barcelona, y de su sector turístico en particular, no puede disociarse de estos factores.

\section{AGRADECIMIENTOS}

El presente artículo se integra dentro del Subprograma Ramón y Cajal del Ministerio de Ciencia e Innovación con la referencia RYC-2008-02456 y del Programa General de Investigación y Desarrollo del Ministerio de Ciencia e Innovación. Referencia SEJ2006-04023.

\section{BIBLIOGRAFIA}

Ajuntament de Barcelona (2005) Marca Barcelona. Barcelona: Departament Promoció Econòmica Ajuntament de Barcelona.

Ashworth G J, y Voogd H (1990) Selling the city: marketing approaches in public sector urban planning. Belhaven Press, London.

Balibrea M P (2006) Barcelona: del modelo a la marca. Forum de cultura, democratizem la democràcia. [Accedido 19 noviembre 2006] http://www.e-barcelona.org

Balibrea M P (2001) Urbanism, culture and the post-industrial city: challenging the "Barcelona model." Journal of Spanish Cultural Studies, 2(2): 187-210.

Bianchini F, Parkinson M (1993) Cultural policy and urban regeneration: the west european experience. Manchester University Press, Manchester.

Bohigas O (1985) Reconstrucció de Barcelona. Edicions 62, Barcelona.

Bohigas O, Buchanan P, Magnano Lampugnani V (1991) Barcelona. City and architecture. Rizzoli, New York.

Brunet F (1995) An economic analysis of the Barcelona's 92 Olympic Games: resources, financing and impact. In Moragas $\mathrm{M}$ de, Botella $\mathrm{M}$ (eds.) The keys to success. The social, sporting, economic and communications impact of Barcelona'92: 203-237.

Capel H (2007) El debate sobre la construcción de la ciudad y el llamado "Modelo Barcelona". Scripta Nova. Revista Electrónica de Geografia y Ciencias sociales, XI (233).

Casellas A (2009) Barcelona's urban landscape: the making of a tourist product. Journal of Urban History, 35(6): 815-832.

Casellas A (2007) Gobernabilidad, participación ciudadana y desarrollo económico: adaptaciones locales a estrategias globales. Scripta Nova. Revista Electrónica de Geografía y Ciencias sociales, XI (243).

Casellas A (2006) Las limitaciones del "modelo Barcelona". Una lectura desde Urban Regime Analysis. Documents d'Anàlisi Geografica, 48: 61-81.

Casellas A (2003) The Barcelona model: agents, policies and planning dynamics in tourism development. Tesis Doctoral. Department of Urban Planning and Policy Development. Rutgers, the State University of New Jersey.

De Delàs I (1999) Director de Planificación y Control de Gestión de Turisme de Barcelona. Entrevista. Enero 11, 1999.

Consell Econòmic i Social de Barcelona (2009) Memòria socioeconòmica de Barcelona 2008. Consell Econòmic i Social de Barcelona, Barcelona.

Consorci Turisme de Barcelona (2009a) Turisme de Barcelona Power Point. Barcelona Turismo, Barcelona. 
Consorci Turisme de Barcelona (2009b) Consorci turisme de Barcelona. Perfil d'empresa, composición, tasca i funcions. Barcelona Turismo, Barcelona.

Edwards D, Griffin T, Hayllar B (2008) Urban tourism research: developing an agenda. Annals of Tourism Research, 35: 1032-1052.

Escolà A (1999) Quarta Tinència d'Alcaldia. Ajuntament de Barcelona. Entrevista, Enero 11, 1999.

Fabre J; Huertas J M (2000) La Barcelona del segle XX. Burguesa i Revolucionària. Flor del Vent, Barcelona.

Florida R (2005) Cities and the creative class. Routledge, London.

Florida R (2002) The rise of the creative class. And how it's transforming work, leisure and everyday life. Basic Books.

Ganau J (2008) Reinventing memories. The origin and development of Barcelona's Barri Gotic 1988-1950. Journal of Urban History: 995-872.

Gold J R, y Gold M M (2008) Olympic cities: regeneration, city rebranding and changing urban agendas. Geography Compass, 2(1): 300-318.

Gospodini A (2001) Urban design, urban space morphology, urban tourism: an emerging paradigm concerning their relationship. European Planning Studies, 9 (7): 925-934.

Holcomb B (1994) City make-overs: marketing the post industrial city. In Gold J, Ward S (eds.) Place promotion: the use of publicity and marketing to sell towns and regions. John Wiley \& Sons, New York: 115-132.

Holcomb B (1993) Revisioning place: de- and re-constructing the image of the industrial city. In Kearns G, Philo C (eds.) Selling places: the city as a cultural capital, past and present. Pergamon Press, New York: 133-143.

Homs J (2010) Departamento Estudios y Estadística, Consorci Turisme de Barcelona. Entrevista, Enero 22, 2001.

Jessop B (2002) Liberalism, neoliberalism and urban governance: A state-theoretical perspective. Antipode, 34(3): 452-472.

Krueger R, Gibbs D (2007) The sustainable development paradox. Urban political economy in the United States and Europe. The Guilford Press, New York.

Law C (1993) Urban tourism: attracting visitors to large cities. Mansell, London.

Lenskyj H J (2002) The best Olympics ever? Social impacts of Sydney 2000. State University of New York Press.

Mackay D (2000) La recuperació del front marítim. Fundació Bosch Gimpera, Universitat de Barcelona (Colección Aula Abierta, Model Barcelona. Quaderns de Gestió, nº 4), Barcelona.

Maragall P (1999) El evento como estrategia del desarollo urbano: Los Juegos Olímpicos del '92. In Maragall P (ed.) Europa próxima. Europa, regiones y ciudades. Edicions Universitat de Barcelona, Barcelona: 249-254.

Marshall T (1990) Barcelona. Planning Practice \& Research. 5(3): 25-28.

Martínez R (2000) El Retorn al centre de la ciutat. Ph.D. Tesis. Departament de Geografia. Universitat de Barcelona.

Martorell J, Bohigas O, Mackay D; Puigdomenech A (1992) La villa olímpica: arquitectura, parques, puerto deportivo. Editorial Gustavo Gili, S.A., Barcelona.

Pallares-Barbera M (2010) Com canviar quan els canvis són difícils? Avantatges i desavantatges de la Barcelona competitiva des de l'economia simbólica. Treballs de la Societat Catalana de Geografia (en prensa).

Payne M (2005) Olympic turnaround: how the Olympic Games stepped back from the brink of extinction to become the world's best known brand-and a multi-billion dollar global franchise. London Business Press, Twyford.

Pareja M, Tapada T (2000) Relating urban changes to social life: two examples at the city centre of Barcelona. Paper presented at the Workshop Urban Renewal and Housing Rehabilitation. Conference on Housing on the $21^{\text {st }}$ Century: Fragmentation and Reorientation. Gävle, Suecia 26-30 Junio 2000.

Perroux F (1961) L'Économie du XXe Siècle. Press Universitaries de France, Paris.

Pla Estratègic de Turisme de la Ciutat de Barcelona (2008) Aspectes crítics $i$ temes clau del turisme a Barcelona. Ajuntament de Barcelona, Barcelona. 
Pla Estratègic de Turisme de Barcelona (1994) Conclusions. Dossier Informatiu. Ajuntament de Barcelona, Barcelona.

Pla Estratègic de Turisme de la Ciutat de Barcelona (2015) http://www.turisme2015bcn.cat/

Preuss H (2004) The economics of staging the Olympics: a comparison of the Games, 1972-2008. Edward Elgar, Cheltenham.

PROMOCIÓ CIUTAT VELLA SA (PROCIVESA) (2002) 14 Anys Tranformant Ciutat Vella. Ajuntament de Barcelona, Barcelona.

Ràfols E (1997) Dinàmiques residencials a la ciutat de Barcelona. Ajuntament de Barcelona. Patronat Municipal de l'Habitatge, Barcelona.

Raventós F (2000) La col.laboració publicoprivada. Aula Barcelona, Barcelona.

Ross H B, Levine M A (2005) Urban politics. Power in metropolitan America. Wadsworth Publishing. Paperback, Wadsworth Publishing.

Sassen S (2006) Cities in a world economy. Thousand Oaks, Pine Forge Press, California.

Tomlinson A (1999) The game's up: essays in the cultural analysis of sport, leisure and popular culture. Ashgate, Aldershot.

Varley A (1992) Barcelona's Olympic facelift. Geographical Magazine, 64: 20-24.

Ward S (1998) Selling places: the marketing and promotion of towns and cities 1850-2000. E \& FN Spon, London.

Zukin S (1992) Postmodern urban landscapes: mapping culture and power. In Lash S, Friedman J (eds.) Modernity and identity. Blackwell, Oxford: 221-247. 